This peer reviewed manuscript has been accepted for publications to the Tribology Letters. Cite this article as: M. Kalin, Aljaz Pogacnik Comparing surface topography parameters of rough surfaces obtained with spectral moments and deterministic methods,Tribology International 93 (2018) 137-141. DOI: https://doi.org/ 10.1016/j.triboint.2015.09.013

\title{
Comparing surface topography parameters of rough surfaces obtained with spectral moments and deterministic methods
}

\author{
M. Kalina, A. Pogačnik ${ }^{a}$, I. Etsion ${ }^{b}$, B. Raeymaekers ${ }^{*}$ \\ aLaboratory for Tribology and Interface Nanotechnology, Faculty of Mechanical Engineering, \\ University of Ljubljana, Bogišičeva 8, 1000 Ljubljana, Slovenia \\ ${ }^{b}$ Department of Mechanical Engineering, Technion - Israel Institute of Technology, Haifa, Israel \\ 'Department of Mechanical Engineering, University of Utah, Salt Lake City, UT, USA
}

Keywords: surface topography; spectral moments; deterministic analysis

\begin{abstract}
Understanding contact between rough surfaces is of critical importance to the design of many engineering applications. Contact models rely on material properties and surface topography of the contacting surfaces as input parameters. Hence, the relevance of the contact models is dependent on their inherent assumptions and the accuracy with which the input parameters are determined. We have evaluated the difference between the surface topography parameters calculated with a statistical and deterministic approach for actual engineering surfaces. We have found topography values that change up to $300 \%$ depending on the method used, and attribute this to the stringent definition of an asperitypeak in the case of deterministic analysis as opposed to statistical analysis, which not only considers asperity-peaks but also asperity-shoulders.
\end{abstract}

Corresponding author: *bart.raeymaekers@utah.edu 
This peer reviewed manuscript has been accepted for publications to the Tribology Letters. Cite this article as: M. Kalin, Aljaz Pogacnik Comparing surface topography parameters of rough surfaces obtained with spectral moments and deterministic methods,Tribology International 93 (2018) 137-141. DOI: https://doi.org/ 10.1016/j.triboint.2015.09.013

\section{Introduction}

Understanding the mechanical interaction between rough surfaces is of critical importance to the design of many engineering applications. Oftentimes, multi-asperity elastic, elastic-plastic, and plastic contact models are used to predict contact parameters such as the real area of contact, normal load, and electrical conductivity as a function of the separation between two contacting rough surfaces $[1,2]$. These contact models rely on material properties and surface topography of the contacting surfaces as input parameters. Hence, the relevance of the contact models is dependent on their inherent assumptions and the accuracy with which the input parameters are determined $[3,4]$. The surface topography of an engineering surface can be determined experimentally using e.g. a stylus profilometer, optical profilometer, or an atomic force microscope (AFM), depending on the size of the specimen or the area that is analyzed, and the desired measurement resolution. The surface is often represented as a matrix of surface heights $z=f(x, y)$, where $z$ is the surface height at coordinates $x$ and $y$. The surface topography of an engineering surface is typically characterized by means of the asperitypeak density $\eta$, mean asperity-peak radius $\rho$, and standard deviation of asperity heights $\sigma_{s}$. Two methods are commonly used to calculate these surface topography parameters from an engineering surface represented as $z=f(x, y)$.

McCool [5] described a statistical method to determine the surface topography parameters of a three-dimensional (3D) isotropic rough surface, based on the spectral moments of a single arbitrary two-dimensional (2D) trace of that rough surface. This method has been widely adopted, see for instance [6-10]. However, realistic engineering surfaces are typically not isotropic as almost all manufacturing techniques result in a surface topography with a preferential direction. Furthermore, it has been shown that the spectral moments may vary significantly for any arbitrary $2 \mathrm{D}$ trace of a rough surface $[3,11]$. To address this problem, several authors have used average values of the spectral moments obtained from a finite number of traces of the 3D surface to calculate the topography parameters $[2,3,11-15]$. 
This peer reviewed manuscript has been accepted for publications to the Tribology Letters. Cite this article as: M. Kalin, Aljaz Pogacnik Comparing surface topography parameters of rough surfaces obtained with spectral moments and deterministic methods, Tribology International 93 (2018) 137-141. DOI: https://doi.org/ 10.1016/j.triboint.2015.09.013

Another commonly used method is based on individually identifying asperity-peaks as local maxima of $z=f(x, y)$ [16-18]. The topography parameters are then calculated directly from these asperity-peaks $[17,18]$ as opposed to relying on statistical methods. This deterministic approach avoids the statistical averaging inherent to the previously described spectral moments approach, and is based on the actual 3D surface topography. Different schemes can be used to identify local maxima, such as the 9 point-peak neighbor [18-20] and the 5 point-peak neighbor $[18,19,21,22]$ schemes. The 9 pointpeak neighbor scheme seems to be the most accurate one [11]. Few works have compared the surface topography parameters calculated with different deterministic methods. Pogacnik and Kalin evaluated the surface topography parameters for 2D profiles [4] and for 3D topography [25] obtained from real engineering surfaces with different roughness. They used a deterministic approach based on three, five, seven and nine neighboring points of a $2 \mathrm{D}$ and $3 \mathrm{D}$ surface characterization and concluded that the choice of the lateral resolution, roughness, and especially the number of neighboring points significantly affects the surface topography parameter results. Pawar et al. [3] calculated the contact parameters of the Greenwood-Williamson (GW) model, based on surface topography parameters obtained with different methods for 3D isotropic numerically generated surfaces. They included McCool's statistical method based on single and multiple traces of the rough surface and the deterministic approach based on a 9 point-peak neighbor scheme in their analysis, and also concluded that the GW contact parameters vary significantly depending on the method that is used to determine the topography parameters, which serve as input to the GW contact model. However, they did not verify their analysis with real engineering surfaces.

Thus, both statistical and deterministic methods suffer from various sources of uncertainty. However, no publications exist that compare these two approaches in a comprehensive way for real engineering surfaces of different surface roughness. Accordingly, the objective of this paper is to evaluate the difference between the surface topography parameters calculated with a spectral moments and deterministic approach. The characterization of surface topography parameters is based on the 
This peer reviewed manuscript has been accepted for publications to the Tribology Letters. Cite this article as: M. Kalin, Aljaz Pogacnik Comparing surface topography parameters of rough surfaces obtained with spectral moments and deterministic methods,Tribology International 93 (2018) 137-141. DOI: https://doi.org/ 10.1016/j.triboint.2015.09.013

experimentally measured 3D engineering surfaces analyzed in $[4,25]$ and not numerically generated surfaces as employed in [3].

\section{Materials and methods}

\subsection{Specimen geometry and surface roughness}

We have used five different stainless steel (100Cr6) specimens, prepared with a sequence of grinding and polishing steps (RotoPol-21, Struers, Denmark) to achieve a distinct average surface roughness, $S_{a}$, ranging from smooth $\left(S_{a}=0.005 \mu \mathrm{m}\right)$ to rough $\left(S_{a}=0.529 \mu \mathrm{m}\right)$. The specimens are manufactured to have an isotropic surface roughness. The hardness of the specimens is $850 \mathrm{HV}_{0.15}$ (62 HRC), measured with a micro-hardness tester (Leitz Miniload, Wild Leitz GmbH, Wetzlar, Germany). An optical interferometer with an additional 20x magnification lens (Contour GT-K0, Bruker, Arizona, USA) is used to measure the surface topography of the specimens over an area of $0.0434 \mathrm{~mm}^{2}$, identical for all specimens to maintain constant pixel size, and with a lateral resolution of $0.187 \mu \mathrm{m}$. Figure 1 shows an optical interferometry image of each of the five specimens, depicting their surface topography. The roughness of each specimen is measured at five randomly selected locations on the specimen to confirm consistent sample preparation. The average and standard deviation of the surface roughness parameters, including the average surface roughness $S_{a}$, the root mean square (RMS) roughness $S_{q}$, the skewness $S_{s k}$, and the kurtosis $S_{k u}$ are calculated and summarized in Table 1. 
This peer reviewed manuscript has been accepted for publications to the Tribology Letters. Cite this article as: M. Kalin, Aljaz Pogacnik Comparing surface topography parameters of rough surfaces obtained with spectral moments and deterministic methods,Tribology International 93 (2018) 137-141. DOI: https://doi.org/ 10.1016/j.triboint.2015.09.013

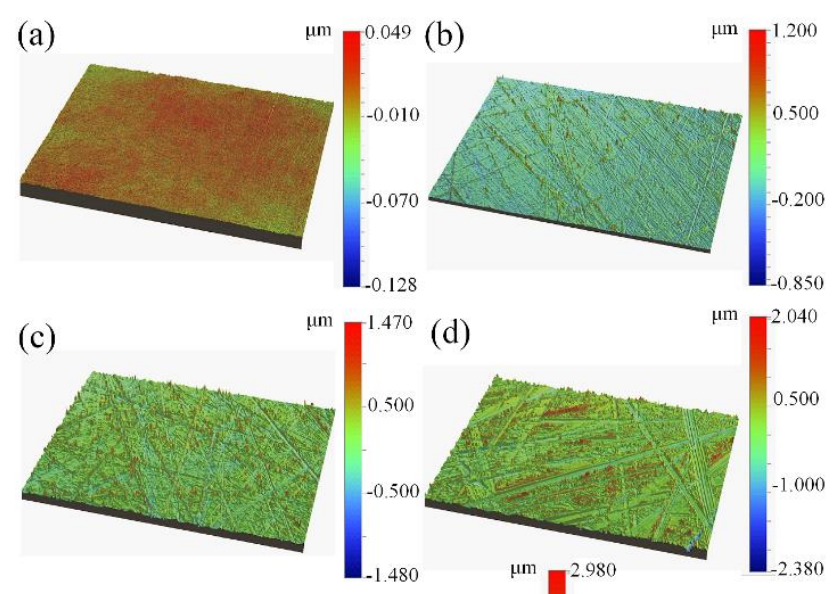

(e)

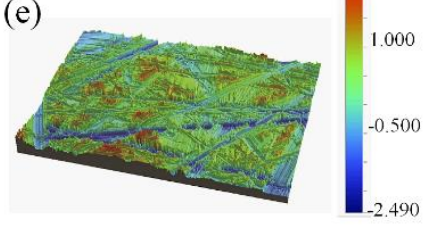

Figure 1: Optical interferometry images of all five specimens, illustrating the surface topography. (a) $S_{a}=0.005 \mu \mathrm{m}$, (b) $S_{a}=0.057 \mu \mathrm{m}$, (c) $S_{a}=0.116 \mu \mathrm{m}$, (d) $S_{a}=0.218 \mu \mathrm{m}$, and (e) $S_{a}=0.529 \mu \mathrm{m}$. The area of the images covers $0.0434 \mathrm{~mm}^{2}$.

Table 1: Average values and standard deviation of $S_{a}, S_{q}, S_{s k}$ and $S_{k u}$ based on five measurements of all five test specimens.

\begin{tabular}{|l|c|c|c|c|}
\hline & $S_{a}[\boldsymbol{\mu m}]$ & $S_{q}[\boldsymbol{\mu m}]$ & $S_{\boldsymbol{s} k}$ & $\boldsymbol{S}_{\boldsymbol{k} u}$ \\
\hline Surface 1 & $0.005 \pm 0.001$ & $0.007 \pm 0.001$ & $3.242 \pm 0.501$ & $12.486 \pm 1.042$ \\
\hline Surface 2 & $0.057 \pm 0.003$ & $0.091 \pm 0.007$ & $2.758 \pm 0.454$ & $10.431 \pm 0.896$ \\
\hline Surface 3 & $0.116 \pm 0.005$ & $0.161 \pm 0.007$ & $1.076 \pm 0.208$ & $7.307 \pm 0.459$ \\
\hline Surface 4 & $0.218 \pm 0.014$ & $0.289 \pm 0.018$ & $0.196 \pm 0.249$ & $4.384 \pm 0.214$ \\
\hline Surface 5 & $0.529 \pm 0.030$ & $0.660 \pm 0.025$ & $0.039 \pm 0.104$ & $2.893 \pm 0.332$ \\
\hline
\end{tabular}

\subsection{Surface topography parameter analysis using the spectral moment method}

The spectral moments $m_{0}, m_{2}$ and $m_{4}$ are defined as [5]:

$m_{0}=A V G\left[\left(z^{2}\right)\right]$ 
This peer reviewed manuscript has been accepted for publications to the Tribology Letters. Cite this article as: M. Kalin, Aljaz Pogacnik Comparing surface topography parameters of rough surfaces obtained with spectral moments and deterministic methods,Tribology International 93 (2018) 137-141. DOI: https://doi.org/ 10.1016/j.triboint.2015.09.013

$$
\begin{aligned}
& m_{2}=A V G\left[\left(\frac{\partial z}{\partial x}\right)^{2}\right] \\
& m_{4}=A V G\left[\left(\frac{\partial^{2} z}{\partial x^{2}}\right)^{2}\right],
\end{aligned}
$$

where AVG represents the arithmetic average and $z(x)$ indicates a 2D trace of the surface heights along an arbitrary $x$-direction of the 3D surface. The asperity-peak density $\eta$, the radius of curvature $\rho$, and the standard deviation of asperity-peak height, $\sigma_{S}$, are then determined as

$$
\begin{aligned}
& \eta=\left(\frac{m_{4}}{m_{2}}\right) / 6 \pi \sqrt{3}, \\
& \rho=0.375\left(\frac{\pi}{m_{4}}\right)^{0.5}, \\
& \sigma_{S}=\left(1-\frac{0.8968}{\alpha}\right)^{0.5} m_{0}^{0.5},
\end{aligned}
$$

where $\alpha=m_{0} m_{4} / m_{2}^{2}$ is the so-called bandwidth parameter. The surface topography data of each specimen, obtained with optical interferometry, consists of 1288 and 966 traces in the $x$ and $y$ direction, respectively, where $x$ and $y$ are two orthogonal directions. We calculate the spectral moments for each trace in both $x$ and $y$-directions and average them for each direction, because $\eta, \rho$, and $\sigma_{S}$ vary considerably when calculated for an arbitrary single $2 \mathrm{D}$ trace $[3,12]$. Furthermore, $\eta, \rho$, and $\sigma_{S}$ depend on the finite difference discretization used to calculate the derivatives in Eqs. (2) and (3) [17]. While several schemes can be used, and the results obtained with different schemes can be related to each other [24], central finite difference discretization is used throughout this work for consistency.

\subsection{Surface topography parameter analysis using the deterministic method}

To determine the asperity-peaks of a 3D rough surface, we have used the deterministic nearest neighbor approach, where an asperity-peak is defined as a point on the $3 \mathrm{D}$ rough surface with a 
This peer reviewed manuscript has been accepted for publications to the Tribology Letters. Cite this article as: M. Kalin, Aljaz Pogacnik Comparing surface topography parameters of rough surfaces obtained with spectral moments and deterministic methods,Tribology International 93 (2018) 137-141. DOI: https://doi.org/ 10.1016/j.triboint.2015.09.013

surface height $z$ larger than its $N$ nearest neighbors. A 5-point peak (5PP-3D) and 9-point peak (9PP3D) criterion identify a point as an asperity-peak if its surface height exceeds that of its 4 or 8 nearest neighbor points, respectively [25]. We calculate the asperity-peak density $\eta$ and the standard deviation of asperity-peak heights $\sigma_{s}$ from all asperity-peaks. The radius of curvature $\rho$ of each individual asperity-peak is calculated as the radius of a sphere that is fitted according to the least-squares method, to the five (or nine) points that define that asperity-peak. A detailed description is available in [25]. We have repeated this procedure for all five measurements of each specimen, and we have calculated the average asperity-peak radius of curvature.

\section{Results}

\subsection{Asperity-peak density}

Figure 2 shows the asperity-peak density calculated with the deterministic and spectral moments analysis, respectively, for five specimens with different surface roughness $S_{a}$. The surface topography of each specimen is measured five times at different, randomly selected locations on the specimen. The bar graph shows the average results of all five measurements, whereas the error bars indicate one standard deviation. Spectral moments analysis in the $x$-direction of the smoothest surface results in an asperity-peak density of $8.4 \mu \mathrm{m}^{-2}$, and the other three results are between $2.1 \mu \mathrm{m}^{-2}$ and $3.3 \mu \mathrm{m}^{-2}$, i.e., as much as four times smaller. The asperity-peak density obtained for the other specimens is between $2.0 \mu \mathrm{m}^{-2}$ and $2.8 \mu \mathrm{m}^{-2}$ for the spectral moments analysis, and between $0.5 \mu \mathrm{m}^{-2}$ and $1.0 \mu \mathrm{m}^{-2}$ for the deterministic analysis. We observe that for the 5PP-3D and 9PP-3D asperity-peak identification criteria, the asperity-peak density is almost constant with increasing surface roughness. The only deviation is found for the smoothest, extremely polished surface. This can be explained because asperity-peaks are generally better defined on a smooth surface with large, curved asperities than on a rough surface with spiky asperities. Hence, more surface points per unit area are higher than their eight nearest neighbors on a smooth compared to a rough surface. The lateral resolution, which determines the distance between adjacent surface points, also plays an important role $[4,20,25]$. Similarly, the 
This peer reviewed manuscript has been accepted for publications to the Tribology Letters. Cite this article as: M. Kalin, Aljaz Pogacnik Comparing surface topography parameters of rough surfaces obtained with spectral moments and deterministic methods, Tribology International 93 (2018) 137-141. DOI: https://doi.org/ 10.1016/j.triboint.2015.09.013

5PP-3D criterion results in a higher number of asperity-peaks than the 9PP-3D criterion, regardless of the surface roughness, because the 9PP-3D criterion is more stringent in terms of its definition of an asperity-peak than the 5PP-3D criterion. The spectral moments analysis shows that the asperity-peak density is almost independent of the direction in which the surface topography is evaluated, because the specimens are manufactured to have an isotropic surface topography. However, for the smoothest surface the analysis in the $x$-direction yields a much higher asperity-peak density than in the $y$ direction, which is explained by the increased difficulty of manufacturing an isotropic surface topography for increasingly smooth surfaces. For all other specimens, which are less smooth (higher $S_{a}$ ), we achieve a more isotropic rough surface. Consequently, the asperity-peak density is independent of the direction of analysis, and is almost independent of the surface roughness. Additionally, the spectral analysis is based on 2D traces that do not necessarily trace asperity-peaks, but possibly also the shoulder of an asperity and, thus, result in a higher apparent asperity-peak density. In contrast, both 3D deterministic approaches [25] impose a stricter definition of an asperity-peak, thus resulting in a lower asperity-peak density. These results for realistic experimentally measured surfaces are in agreement with the findings documented in [3] for numerically generated surfaces, i.e., for numerically generated isotropic rough surfaces the asperity-peak density increases with increasing surface roughness, and the spectral moments method predicts an asperity-peak density that is significantly higher than that predicted by the deterministic approach.




This peer reviewed manuscript has been accepted for publications to the Tribology Letters. Cite this article as: M. Kalin, Aljaz Pogacnik Comparing surface topography parameters of rough surfaces obtained with spectral moments and deterministic methods,Tribology International 93 (2018) 137-141. DOI: https://doi.org/ 10.1016/j.triboint.2015.09.013

Figure 2: Asperity-peak density of five specimens with different average surface roughness $S_{a}$ for the deterministic and spectral moments analyses. The results show the average of five measurements and the error bars indicate one standard deviation.

\subsection{Asperity-peak radii}

Figure 3 shows the asperity-peak radius calculated with the deterministic and spectral moments analyses, respectively, for five specimens with different surface roughness $S_{a}$. The bar graph shows the average results of five surface topography measurements, whereas the error bars indicate one standard deviation. We observe that with both analyses the average asperity-peak radius decreases with increasing surface roughness. The average asperity-peak radius calculated using the spectral moments method is significantly lower than that obtained when using a deterministic approach, for all specimens except for the smoothest surface in the $y$-direction, where spectral moments analysis yields an average asperity-peak radius double that of the three other results. However, in general, the difference between spectral analysis in $x$ and $y$-direction is negligible. The average asperity-peak radius calculated using the 5PP-3D criterion and the $9 \mathrm{PP}-3 \mathrm{D}$ criterion is just slightly lower for the former. This is especially true for $S_{\mathrm{a}} \geq 0.116 \mu \mathrm{m}$, where the asperity radius is almost independent of the surface roughness. For the deterministic analysis the mean asperity radius is found to be between 1 and $2 \mu \mathrm{m}$, whereas for the spectral moments analysis this is between 0.1 and $0.5 \mu \mathrm{m}$. An exception is again the extremely smooth surface $\left(S_{a}=5 \mathrm{~nm}\right)$, which, however, is non-typical for the majority of engineering applications.




This peer reviewed manuscript has been accepted for publications to the Tribology Letters. Cite this article as: M. Kalin, Aljaz Pogacnik Comparing surface topography parameters of rough surfaces obtained with spectral moments and deterministic methods,Tribology International 93 (2018) 137-141. DOI: https://doi.org/ 10.1016/j.triboint.2015.09.013

Figure 3: Asperity-peak radius of five specimens with different roughness parameter $S_{a}$ for the deterministic and spectral moments analysis. The results show the average of five measurements and the error bars indicate one standard deviation.

\subsection{Asperity-peak height standard deviation}

Figure 4 shows the standard deviation of asperity-peak heights calculated with the deterministic and spectral moments analyses, respectively, as a function of the five specimens with different surface roughness $S_{a}$. The bar graph shows the average results of five surface topography measurements, whereas the error bars indicate one standard deviation. We observe that the standard deviation of asperity-peak heights increases almost linearly with increasing surface roughness $\left(R^{2}>0.87\right)$, from $0.006 \mu \mathrm{m}$ for a smooth surface to $0.7 \mu \mathrm{m}$ for a rough surface, except for the smoothest surface where the standard deviation calculated with both methods is almost identical. The deterministic analysis always results in a higher standard deviation of asperity-peak heights compared to the spectral moments analysis, independent of the direction in which the spectral moments are calculated. The deterministic analysis in 3D is more restrictive and accounts for clear and better defined asperity-peaks, whereas the spectral moments approach relies on a $2 \mathrm{D}$ trace of the surface, which may also trace asperity shoulders rather than peaks and, thus, results in a lower standard deviation of asperity-peak heights. This is in agreement with Fig. 2, where a higher asperity-peak density is observed for the spectral moments approach than for the deterministic approach. 
This peer reviewed manuscript has been accepted for publications to the Tribology Letters. Cite this article as: M. Kalin, Aljaz Pogacnik Comparing surface topography parameters of rough surfaces obtained with spectral moments and deterministic methods,Tribology International 93 (2018) 137-141. DOI: https://doi.org/ 10.1016/j.triboint.2015.09.013



Figure 4: Standard deviation of asperity-peak height of five specimens with different roughness parameter $S_{a}$ for the deterministic and spectral moments analysis. The results show the average of five measurements and the error bars indicate one standard deviation.

\section{Conclusions}

On the basis of the experimental analysis of five polished engineering surfaces with average roughness $0.005 \leq S_{a} \leq 0.529 \mu \mathrm{m}$, the following conclusions are made:

1. Extremely smooth surfaces (in our study $S_{a}=5 \mathrm{~nm}$ ) are a source of inconsistencies in surface analyses both for deterministic and statistical analyses.

2. The asperity-peak density for the surfaces used in this study is calculated to be between $0.5 \mu \mathrm{m}^{-2}$ and $1 \mu \mathrm{m}^{-2}$ with the deterministic approach, and between $2 \mu \mathrm{m}^{-2}$ and $2.5 \mu \mathrm{m}^{-2}$ with the statistical approach, independent of the direction in which it is measured. Asperity-peak density is found to be almost independent of the surface roughness $S_{a}$ for $S_{a}>0.05 \mu \mathrm{m}$, when determined with the deterministic approach, and increase slightly with increasing surface roughness when determined using the statistical approach.

3. The average asperity-peak radius for the surfaces used in this study is calculated to be between $1 \mu \mathrm{m}$ and $2 \mu \mathrm{m}$ with the deterministic approach, and between $0.1 \mu \mathrm{m}$ and $0.5 \mu \mathrm{m}$ with the 
This peer reviewed manuscript has been accepted for publications to the Tribology Letters. Cite this article as: M. Kalin, Aljaz Pogacnik Comparing surface topography parameters of rough surfaces obtained with spectral moments and deterministic methods,Tribology International 93 (2018) 137-141. DOI: https://doi.org/ 10.1016/j.triboint.2015.09.013

statistical approach. It decreases slightly with increasing surface roughness, but this observation becomes insignificant for $S_{a}>0.116 \mu \mathrm{m}$.

4. The standard deviation of asperity-peak heights increases almost linearly with increasing surface roughness for both statistical and deterministic approaches. However, it is typically higher for the deterministic than for statistical method. The difference between the two methods decreases with increasing surface roughness; from approximately $40 \%$ to $5 \%$ in our study.

5. The reason for observing a larger asperity-peak density and a lower standard deviation of asperity-peak heights with the statistical compared to the deterministic approach results from a less restrictive definition of an asperity-peak.

\section{Acknowledgement}

A.P. and M.K. acknowledge partial financial support of the European Social Fund.

\section{References}

[1] G. Carbone, F. Bottiglione, Contact mechanics of rough surfaces: a comparison between theories, Meccanica 46: 557-565 (2011)

[2] R.L. Jackson, I. Green, On the modeling of elastic contact between rough surfaces, Trib. Trans. 54: 300-314 (2011)

[3] G. Pawar, P. Pawlus, I. Etsion, B. Raeymaekers, The effect of determining topography parameters on alayzing elastic contact between isotropic rough surfaces, J. Tribol. T.-ASME 135(1): 011401 (2013)

[4] A. Pogacnik, M. Kalin, How to determine the number of asperity peaks, their radii and their heights for engineering surfaces: a critical appraisal, Wear 300: 143-154 (2013)

[5] J.I. McCool, Relating profile instrument measurements to the functional performance of rough surfaces, J. Tribol. T.-ASME 109(2): 264-270 (1987)

[6] I. Etsion, M. Amit, The effect of small normal loads on the static friction coefficient for very smooth surfaces, J. Tribol. T.-ASME 115(3): 406-410 (1993)

[7] L. Kogut, R.L. Jackson, A comparison of contact modeling utilizing statistical and fractal approaches, J. Tribol. T.-ASME 128(1): 213-217 (2006)

[8] C.H. Lee, A.A. Polycarpou, Static friction experiments and verification of an improved elastic-plastic model including roughness effects, J. Tribol. T.-ASME 129(4): 754-760 (2007)

[9] S. Akbarzadeh, M. Khonsari, Performance of spur gears considering surface roughness and shear thinning lubricant, J. Tribol. T.-ASME 130(2): 021503 (2008)

[10] C.H. Lee, M. Eriten, A.A. Polycarpou, Application of elastic-plastic static friction models to rough surfaces with asymmetric asperity distribution, J. Tribol. T.-ASME 132(3): 031602 (2010) 
This peer reviewed manuscript has been accepted for publications to the Tribology Letters. Cite this article as: M. Kalin, Aljaz Pogacnik Comparing surface topography parameters of rough surfaces obtained with spectral moments and deterministic methods,Tribology International 93 (2018) 137-141. DOI: https://doi.org/ 10.1016/j.triboint.2015.09.013

[11] R.S. Sayles, T.R. Thomas, Measurements of the statistical microgeometry of engineering surfaces, J. Lubr. Technol. 101: 409-417 (1979)

[12] J.I. McCool, Predicting microcontact in ceramics via a microcontact model, J. Tribol. T.ASME 108(3): 380-386 (1986)

[13] M. Uchidate, A. Iwabuchi, K. Kikuchi, T. Shimizu, Research on the validity of using nayak's theory for summit parameters of discrete isotropic Gaussian surfaces, J. Adv. Mech. Des. Syst. Manuf. 3(2): 125-135 (2009)

[14] R.D.I. Dickey, R. Jackson, G. Flowers, Measurements of the static friction coefficient between tin surfaces and comparison to a theoretical model, J. Tribol. T.-ASME 133(3): 031408 (2011)

[15] B. Raeymaekers, I. Etsion, F.E. Talke, Enhancing tribological performance of the magnetic tape/guide interface by laser surface texturing, Tribol. Lett. 27(1): 89-95 (2007)

[16] J.A. Greenwood, A unified theory of surface roughness, P. Roy. Soc. A. Math. Phys., 393: 133-157 (1984)

[17] E. Tomanik, H. Chacon, G. Teixeira, A simple numerical procedure to calculate the input data of Greenwood-Williamson model of asperity contact for actual engineering surfaces, Tribology S. 41: 205-215 (2003)

[18] N. Yu, A.A. Polycarpou, Extracting summit roughness parameters from random Gaussian surfaces accounting for asymmetry of the summit heights, J. Tribol. T.-ASME 126(4): 761766 (2004)

[19] P. Pawlus, Digitisation of surface topography measurement results, Measurement 40(6): 672686 (2007)

[20] A. Suh, A.A. Polycarpou, T. Conry, Detailed surface roughness characterization of engineering surfaces undergoing tribological testing leading to scuffing, Wear 225(1-6): 556$568(2003)$

[21] M. Li, M.J. Phillips, D.J. Whitehouse, Extension of two-dimensional sampling theory, J. Phys. A. Math. Gen. 22: 5053-5063 (1989)

[22] C.Y. Poon, B. Bhushan, Comparison of surface roughness measurements by stylus profiler, AFM and non-contact optical profiler, Wear 190(1): 76-88 (1995)

[23] J.A. Greenwood, J.J. Wu, Surface roughness and contact: an apology, Meccanica 36: 617-630 (2001)

[24] M. Paggi, M. Ciavarella, The coefficient of proportionality $\kappa$ between real contact area and load, with new asperity models, Wear 268: 1020-1029 (2010)

[25] M. Kalin, A. Pogacnik, Criteral and properties of the asperity peaks on 3D engineering surfaces, Wear 308: 95-104 (2013) 